\title{
Valores ecobiométricos e índice de resistividade da artéria oftálmica externa em catetos (Tayassu tajacu, Linnaeus, 1758)
}

[Ecobiometric values and resistivity index of the external ophthalmic artery in collared peccaries (TayassutajacuLinnaeus, 1758)]

\author{
S.B. Araújo ${ }^{1}$, F.R. Alves ${ }^{2}$, G.T. Pessoa ${ }^{3}$, R.P.S. Rodrigues ${ }^{4}$, L.S. Moura $^{4}$, \\ A.B.S. Silva ${ }^{4}$, A.P. Souza ${ }^{1}$
}

\author{
${ }^{1}$ Programa de pós-graduação - Universidade Federal de Campina Grande - Campus de Patos, PB \\ ${ }^{2}$ Universidade Federal do Piauí - Teresina, PI \\ ${ }^{3}$ Centro Universitário Maurício de Nassau - Teresina, PI \\ ${ }^{4}$ Aluno de pós-graduação - Universidade Federal do Piauí - Teresina, PI
}

\begin{abstract}
RESUMO
Foram avaliados ultrassonograficamente, pela via transpalpebral, 28 bulbos oculares de 14 catetos adultos, através de técnica padronizada pelo operador. Adicionalmente foi realizado o estudo hemodinâmico da artéria oftálmica externa pela técnica de Doppler colorido. Os dados coletados foram analisados estatisticamente pelo programa Bioestat 5.0 for Windows, adotando-se $5 \%$ de significância. Com a metodologia empregada, obtiveram-se os seguintes valores para os globos oculares direito e esquerdo, respectivamente D1: $1,72 \pm 0,29 \mathrm{~mm}$ e $1,76 \pm 0,40 \mathrm{~mm}$; D2: $9,95 \pm 1,08 \mathrm{~mm}$ e 10,6 $\pm 0,99 \mathrm{~mm}$; D3: 7,42 \pm $0,93 \mathrm{~mm}$ e $7,45 \pm 0,72 \mathrm{~mm}$ e D4: $17,6 \pm 0,78 \mathrm{~mm}$ e $17,8 \pm 0,59 \mathrm{~mm}$. Os valores médios do índice de resistividade da artéria oftálmica externa foram $0,435 \pm 0,02$ e $0,448 \pm 0,02$ (globos oculares direito e esquerdo, respectivamente). Não houve diferença estatística quanto aos antímeros oculares em nenhum dos parâmetros estudados. Conclui-se que a ecobiometria ocular e a Dopplerfluxometria da artéria oftálmica na espécie Tayassu tajacu é executável e reprodutível, desde que haja domínio do examinador em relação à anatomia e à técnica adequada. Os valores inferidos neste estudo servem de referência para médicos veterinários no diagnóstico de doenças oculares.
\end{abstract}

Palavras-chave: porco do mato, olho, hemodinâmica, ultrassonografia

\begin{abstract}
Twenty-eight ocular bulbs of fourteen adult catheters were evaluated through ultrasound with the transpalpebral approach, using a standardized technique by the operator. Additionally, the hemodynamic study of the external ophthalmic artery was performed using the color Doppler technique. The collected data were statistically analyzed by the Bioestat 5.0 for Windows program, adopting $5 \%$ of significance. With the methodology employed, the following values were obtained for the right and left eyeballs, respectively D1: $1.72 \pm 0.29 \mathrm{~mm}$ and $1.76 \pm 0.40 \mathrm{~mm} ; \mathrm{D2}: 9.95 \pm 1.08 \mathrm{~mm}$ and $10.6 \pm 0.99 \mathrm{~mm} ; \mathrm{D3}: 7.42 \pm$ $0.93 \mathrm{~mm}$ and $7.45 \pm 0.72 \mathrm{~mm}$ and $D 4: 17.6 \pm 0.78 \mathrm{~mm}$ and $17.8 \pm 0.59 \mathrm{~mm}$. The mean resistivity index values of the external ophthalmic artery were $0.435 \pm 0.02$ and $0.448 \pm 0.02$ (right and left eyeball respectively). There was no statistical difference regarding ocular antimers in any of the studied parameters. We concluded that ocular echobiometry and ophthalmic artery Doppler flowmetry in Tayassu tajacu species is executable and reproducible, provided there is an examiner's domain regarding the anatomy and proper technique. The values inferred in this study serve as a reference for veterinarians in the diagnosis of eye diseases.
\end{abstract}

Keywords: bush pig, eye, hemodynamics, ultrasound

Recebido em 19 de setembro de 2019

Aceito em 17 de junho de 2020

E-mail: sabrina_vet@yahoo.com.br 


\section{INTRODUÇÃO}

A visão é um sentido crucial para a sobrevivência dos animais, principalmente os de vida livre, dessa forma alterações na acuidade visual podem dificultar ou impossibilitar a busca por alimentos, a locomoção e a defesa contra predadores. Com isso, a oftalmologia veterinária preocupa-se constantemente em promover uma visão o mais próximo possível da emetropia, buscando melhorar a qualidade de vida das espécies (Monteiro e Allemann, 2001; Harrington et al., 2013; Barros et al., 2016). Atualmente, existem inúmeras técnicas para diagnósticos de patologias oftálmicas, entre elas a ultrassonografia que utiliza os ecos produzidos pelas reflexões das ondas sonoras para análise de estruturas. Pela sua composição, o globo ocular é uma excelente interface acústica para estudos ultrassonográficos, permitindo a detecção de diversas alterações (Monteiro e Allemann, 2001; Carvalho et al., 2009).

A ecobiometria ocular foi descrita em espécies domésticas e em algumas silvestres, tais como chinchilas, gatos, coelhos, corujas, capivaras e macacos bugio-ruivo (Montiani-Ferreira et al., 2008; Lima et al., 2010; Squarzoni et al., 2010; Toni et al., 2010; Mirshahi et al., 2014; Souza et $a l ., 2018)$. Soma-se aos estudos ecobiométricos a utilização do Doppler colorido, que é de grande interesse por permitir estudos hemodinâmicos, como condições de fluxo e mensurações de velocidade, caracterizando a impedância vascular (Diniz et al., 2004; Carvalho et al., 2009).

Tendo em vista a sensibilidade e a importância da ultrassonografia, dentro do contexto acima apresentado, objetivou-se com este estudo tanto verificar se a ultrassonografia em modo B pela via transpalpebral permite a identificação das estruturas anatômicas oculares, avaliando a presença de artefatos e de fatores limitantes, quanto determinar valores presumidamente normais da ecobiometria e do índice de resistividade da artéria oftálmica externa em catetos (Tayassu tajacu).

\section{MATERIAL E MÉTODOS}

O estudo foi conduzido após autorização da Comissão de Ética no Uso de Animais (Ceua) da Universidade Federal do Piauí (UFPI), protocolo n ${ }^{\circ} 467 / 18$, e aprovação do Instituto Chico Mendes de Conservação da Biodiversidade (ICMBIO), por meio do Sistema de Autorização e Informação em Biodiversidade (Sisbio), protocolo n ${ }^{\circ} 61038$ 1. Os animais utilizados foram provenientes do Núcleo de Estudos e Preservação de Animais Silvestres (Nepas) do Centro de Ciências Agrárias (CCA)/UFPI (registro $n^{\circ}$ 02/08-618). No Nepas, os animais são alojados em baias coletivas, recebem diariamente dieta formulada pelo veterinário responsável e ingerem água ad libitum.

Para a realização dos exames, os catetos foram capturados com auxílio de puçá e mantidos em baias para realização do jejum alimentar de 12 horas e do jejum hídrico de duas horas, para a posterior sedação. Após o período estimado, foi realizada a contenção química dos animais com aplicação, por via intramuscular, de uma associação de cloridrato de cetamina em solução a $5 \%(15 \mathrm{mg} / \mathrm{kg})$ e midazolan $(1 \mathrm{mg} / \mathrm{kg})$, como preconizado na literatura (Moura et al., 2019). A ação dos fármacos ocorreu cerca de 10 minutos após a aplicação e teve uma duração média de 30 a 40 minutos, sem necessidade de reaplicação ou intercorrências.

Sedados, os animais foram levados para o Núcleo de Diagnóstico por Imagem Veterinário Especializado (Nudive) do CCA/UFPI, para a realização da coleta dos dados da pesquisa; apenas animais hígidos e fêmeas não prenhes participaram do estudo. Foram utilizados 28 bulbos oculares de 14 animais da espécie Tayassu tajacu, sendo seis machos e oito fêmeas.

A técnica ultrassonográfica empregada foi pela via transpalpebral (Carvalho et al., 2009), com um transdutor convexo multifrequencial $5-8 \mathrm{MHz}$ (C8-5), conectado a aparelho de ultrassonografia (Philips ${ }^{\circledR}$ HD15). Todos os registros foram iniciados pelo bulbo ocular esquerdo, e as medidas realizadas pelo mesmo examinador. Após identificar as estruturas, foram mensuradas as distâncias em milímetros ( $\mathrm{mm}$ ) da espessura da câmara anterior (distância entre a córnea e a cápsula anterior do cristalino - D1), espessura do cristalino (distância entre a cápsula anterior e posterior do cristalino - D2), espessura da câmara vítrea (distância entre a cápsula posterior do cristalino e a interface vitreorretiniana - D3) e o comprimento axial do bulbo ocular (distância entre a córnea e a interface vitreorretiniana-D4). Os valores das medidas foram obtidos por meio de 
três mensurações consecutivas em cada olho (Fig. $1)$.

A artéria oftálmica externa foi identificada por coloração Doppler na topografia do nervo óptico, e um ângulo paralelo ao fluxo foi usado para obter o índice de resistividade. Foram ajustadas as escalas de velocidade, adequando as frequências de repetição de pulso para posterior aquisição do espectro de onda Doppler. Todas as medidas foram calculadas automaticamente pelo software próprio do equipamento de ultrassonografia (Fig. 2). Os dados coletados foram analisados no programa Bioestat 5.0 for Windows. Inicialmente foi testada a normalidade por meio do teste de Shapiro-Wilk, para cada parâmetro; posteriormente, foi realizado o teste $\mathrm{T}$ pareado, adotando-se um nível de significância de $5 \%$ $(\mathrm{P}<0,05)$

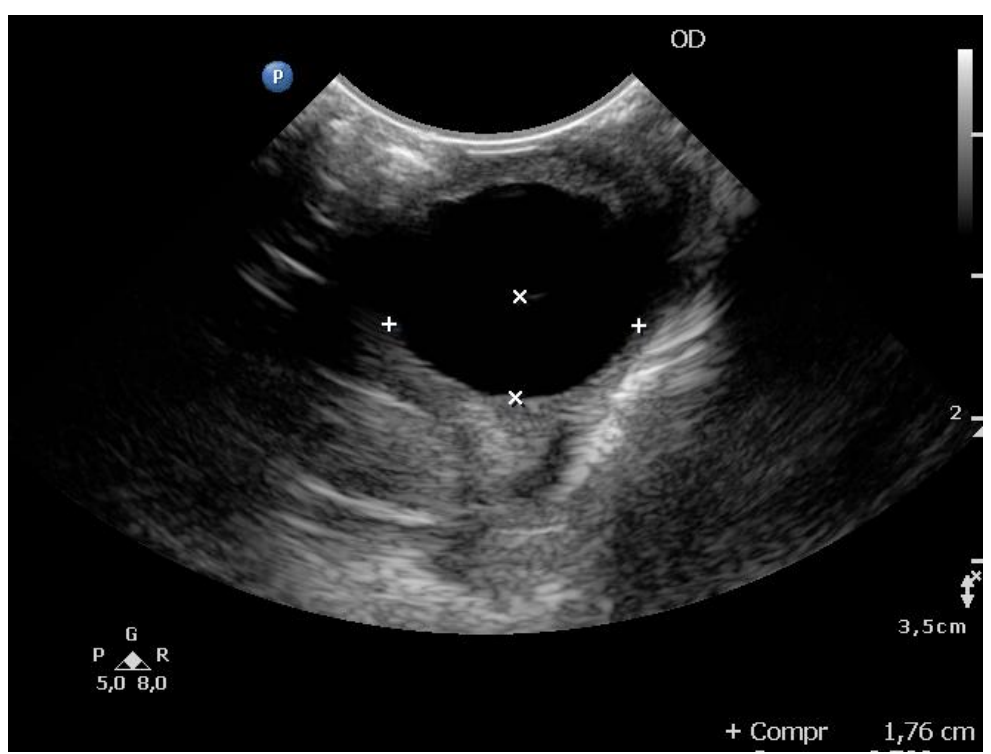

Figura 1. Imagem ultrassonográfica em modo-B representando as características do bulbo ocular da espécie Tayassu tajacu. Marcadores indicam a espessura e o comprimento da câmara vítrea.

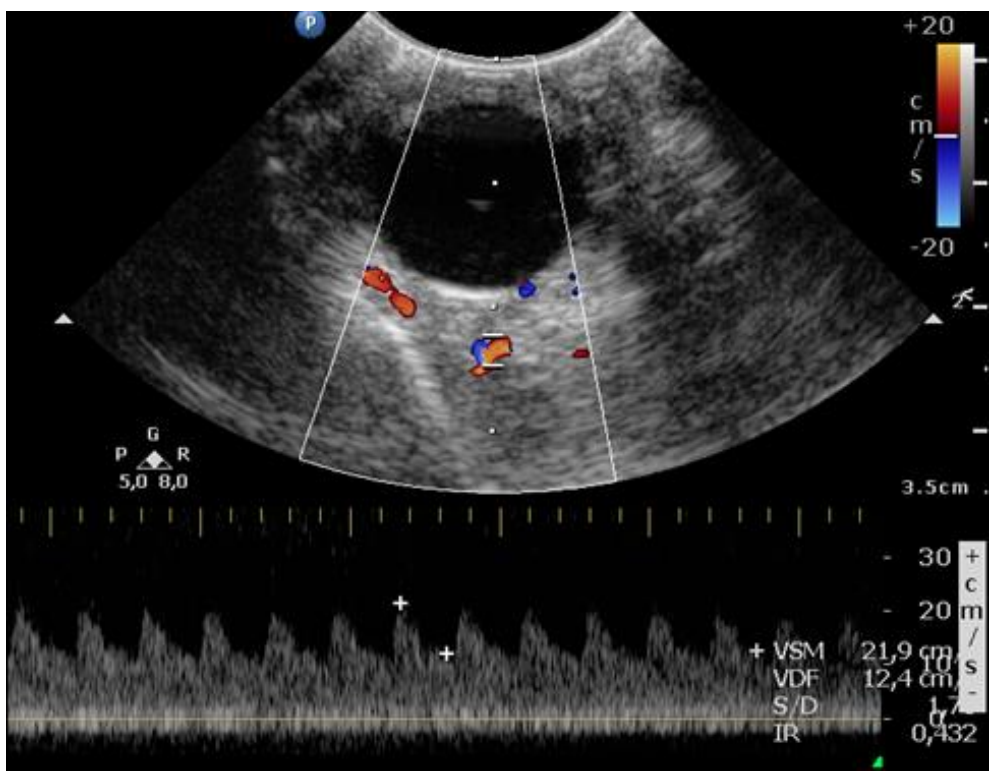

Figura 2. Imagem ultrassonográfica em modo B representando as características do fluxo vascular da artéria oftálmica externa para obtenção do valor do índice de resistividade na espécie Tayassu tajacu 


\section{RESULTADOS}

O protocolo utilizado para a contenção química foi satisfatório, permitindo a execução do exame sem intercorrências. Dez minutos após a administração da associação dos fármacos, os animais semirrotacionaram o globo ocular, sendo necessárias a execução de manobras de angulação do transdutor para obtenção de imagens com qualidade satisfatória. Os dados obtidos com o exame ultrassonográfico ocular dos catetos (Tayassu tajacu) apresentaram distribuição normal pelo teste de Shapiro-Wilk. No teste T pareado para as medidas oculares, não houve diferença estatística nos valores biométricos para os antímeros direito e esquerdo. A Tab. 1 descreve os valores da ecobiometria ocular obtidos. A correlação do comprimento axial com a espessura da lente resultou numa relação de 1:1,76.

Os testes estatísticos empregados (Shapiro-Wilk e $\mathrm{T}$ pareado) não demonstraram diferença entre os globos oculares direito e esquerdo em relação aos parâmetros de Dopplerfluxometria e índice de resistividade da artéria oftálmica (Tab. 2), com valor de t crítico bilateral maior do que o nível de significância $(0,05)$.

Tabela 1. Valores médios, desvio-padrão (DP), valor de p (bilateral) e intervalo de confiança (IC 95\%) para as medidas dos olhos direito e esquerdo de catetos (Tayassu tajacu), em milímetros (mm), espessura da câmara anterior (D1), espessura do cristalino (D2), espessura da câmara vítrea (D3) e comprimento axial (D4), obtidos por meio de ultrassonografia bidimensional, Teresina, PI, Brasil

\begin{tabular}{cccc}
\hline & Variável & Globo ocular direito & Globo ocular esquerdo \\
\hline \multirow{4}{*}{ D1 } & Média & $1,72 \mathrm{~mm}$ & $1,76 \mathrm{~mm}$ \\
& DP & 0,29 & 0,40 \\
D2 & 0,79 & \\
& IC $95 \%$ & $-0,31 \mathrm{a} 0,24$ & $10,6 \mathrm{~mm}$ \\
& Média & $9,95 \mathrm{~mm}$ & 0,99 \\
D3 & DP & 1,08 & \\
& p & 0,09 & $7,45 \mathrm{~mm}$ \\
& IC 95\% & $1,48 \mathrm{a} 0,13$ & 0,72 \\
& Média & $7,42 \mathrm{~mm}$ & \\
& DP & 0,93 & $17,8 \mathrm{~mm}$ \\
D4 & p & 0,94 & 0,59 \\
& IC 95\% & $-0,67 \mathrm{a} 0,62$ & \\
& Média & $17,6 \mathrm{~mm}$ & \\
\hline
\end{tabular}

Tabela 2. Valores médios do índice de resistividade (IR) da artéria oftálmica externa direita e esquerda de catetos (Tayassu tajacu), Teresina, PI, Brasil

\begin{tabular}{|c|c|c|}
\hline & $\begin{array}{c}\text { Globo ocular } \\
\text { direito }\end{array}$ & $\begin{array}{c}\text { Globo ocular } \\
\text { esquerdo }\end{array}$ \\
\hline Média & 0,435 & 0,448 \\
\hline DP & 0,02 & 0,02 \\
\hline $\mathrm{p}$ & 0,19 & \\
\hline IC $95 \%$ & - 0,034 a 0,007 & \\
\hline
\end{tabular}

\section{DISCUSSÃO}

A ultrassonografia ocular é uma técnica simples, não invasiva e eficaz, na caracterização de algumas enfermidades intra e extraoculares.
Segundo a literatura, são preferíveis transdutores com alta frequência, devido à disposição anatômica superficial das estruturas oculares. Neste estudo, a frequência de $8 \mathrm{MHz}$ permitiu a realização da ecobiometria nos catetos. Uma frequência similar $(7,5 \mathrm{MHz})$ foi utilizada na caracterização do olho da capivara, não sendo descritas pelos autores limitações na qualidade e resolução para as mensurações ecobiométricas na espécie supracitada (Montiani-Ferreira et al., 2008). Os dados da presente pesquisa foram obtidos por meio de mensuração direta das estruturas oculares, pelo mesmo examinador, seguindo-se uma metodologia única para todos os animais. 
Como protocolo para a contenção química dos catetos, utilizou-se o cloridrato de cetamina e o midazolan. $\mathrm{Na}$ literatura, é descrito que essa associação produz um efeito que permite a contenção adequada com facilidade de manipulação. Além disso, esses fármacos são constantemente empregados na anestesia e como medicação pré-anestésica de animais de pequeno porte, por proporcionarem segurança, mínimo efeito depressor e rápida recuperação (Moura et al., 2019). Como efeito adverso do protocolo empregado, os catetos semirrotacionaram o globo ocular, sendo necessárias manobras de angulação do feixe sonoro do transdutor para a obtenção de imagens com qualidade satisfatória para as mensurações.

Em um estudo comparativo entre a contenção por método físico com a utilização do puçá e a contenção por método químico com dardos anestésicos com solução de cetamina, xilazina e diazepan em catetos, concluiu-se que a tranquilização química reduz os parâmetros indicadores de estresse e facilita o manejo dos animais. $\mathrm{Na}$ realização de estudos ultrassonográficos, a contenção física é inviável, pois há riscos inerentes ao temperamento do animal, bem como a impossibilidade de eles permanecerem imóveis durante a realização do exame; contudo, o protocolo anestésico descrito pelos autores no estudo pode ser uma opção na realização de ultrassonografia oftálmica em catetos, tendo em vista que foi seguro, e não são descritas alterações no posicionamento do globo ocular (Batista et al., 2009).

No presente estudo, foram incluídos catetos machos e fêmeas adultos, examinando-se os dois olhos. Isso permitiu a determinação de possíveis variabilidades dos resultados, quanto ao sexo e à lateralidade, o que não foi encontrado, corroborando os achados descritos na literatura (Squarzoni et al., 2010; Toni et al., 2010; Barros et al., 2016).

Quanto ao comprimento axial na oftalmologia humana, os olhos podem ser classificados em extremamente curtos (comprimento axial abaixo de $18 \mathrm{~mm}$ ) e curtos (comprimento axial de $18 \mathrm{~mm}$ a 22mm) (Narvaéz et al., 2006). Ao atribuir esse critério para os catetos, nota-se que seus olhos são extremamente curtos, similares aos olhos do miniporco e do coelho (Toni et al., 2010; Barros et al., 2016). Corujas $(24 \pm 0,86 \mathrm{~mm})$, capivaras
$(22,10 \pm 1,71 \mathrm{~mm})$, felinos $(20,91 \pm 0,53 \mathrm{~mm}) \mathrm{e}$ cães $(20,43 \pm 1,48 \mathrm{~mm})$ possuem olhos curtos (Gaiddon et al., 1991; Gilger et al., 1998; Montiani-Ferreira et al., 2008; Squarzoni et al., 2010).

Os valores da espessura da câmara anterior dos catetos foram $1,72 \pm 0,29 \mathrm{~mm}$ e $1,76 \pm 0,40 \mathrm{~mm}$ (globos oculares direito e esquerdo, respectivamente), não existindo diferença entre os antímeros. Nas mensurações oculares de macacos rhessus adultos foram encontrados valores médios superiores aos dos catetos, divergência que pode ser justificada pela maior dimensão corporal e maior peso dessa espécie (Fernandes et al., 2003). A espessura do cristalino dos catetos foi 9,95 \pm $1,08 \mathrm{~mm}$ e $10,6 \pm 0,99 \mathrm{~mm}$ (globos oculares direito e esquerdo, respectivamente), inferior aos valores encontrados em seres humanos (4,24 $\pm 0,06 \mathrm{~mm})$ e capivaras $(7,41 \pm 0,7 \mathrm{~mm})$; já em diferentes espécies de cervídeos da fauna brasileira (média $10 \mathrm{~mm}$ ), são descritos valores similares aos encontrados neste estudo (Hoffer, 1980; Montiani-Ferreira et al., 2008; Crivelaro, 2014).

A espessura da câmara vítrea dos catetos foi 7,42 $\pm 0,93 \mathrm{~mm}$ e $7,45 \pm 0,72 \mathrm{~mm}$ (olhos direito e esquerdo, respectivamente), valor inferior ao de miniporcos, que, embora apresentem um menor porte, possuem uma média superior $(9,39 \pm$ $0,55 \mathrm{~mm}$ ) para esse segmento do bulbo ocular (Barros et al., 2016). Ao se correlacionar a espessura da lente com o comprimento axial do globo ocular em catetos, nota-se uma relação de $1: 1,76$, o que lhes confere a característica anatômica de olhos pequenos com uma lente grande, assim como se observa em roedores (Mutti et al., 1998; Bantseev et al., 2004; Lima et al., 2010).

Essa lente com um formato mais esférico aumenta o poder óptico especialmente para perto, pois há uma redução da tração sofrida pelos ligamentos suspensores causada pela contração dos músculos ciliares. Trata-se de uma particularidade importante, pois aumenta a visibilidade, especialmente na procura por alimentos. $\mathrm{Na}$ medicina veterinária são escassos dados sobre a anatomia vascular do bulbo ocular, dessa forma este estudo seguiu as orientações anatômicas ultrassonográficas de trabalhos realizados em humanos (Diniz et al., 2004). 
A caracterização ultrassonográfica da artéria oftálmica externa em catetos foi dificultosa em razão da semirrotação do globo ocular. Foram necessárias manobras de posicionamento do transdutor para a obtenção de um ângulo adequado para as mensurações. No bugio-ruivo (Alouatta fusca), os pesquisadores também relataram dificuldade na obtenção do traçado espectral da artéria oftálmica (Souza et al., 2018). Nos catetos, essa artéria demonstrou uma baixa resistência, o que já era esperado por se tratar de um vaso com fino calibre. Os valores do índice de resistividade da artéria oftálmica externa nos catetos foram $0,435 \pm 0,02$ e 0,448 $\pm 0,02$ (globos oculares direito e esquerdo, respectivamente). Em macacos-prego $(0,71 \pm 0,03)$, são relatados valores superiores para esse parâmetro (Rodas, 2014).

Nos seres humanos, é descrita uma correlação entre a idade e o fluxo orbital, devido ao aumento da pressão arterial, à diminuição do lúmen ou ao enrijecimento da parede de vasos peribulbares (Williamson et al., 1995; Diniz et al., 2004). Neste estudo, todos os catetos apresentaram uma onda forte, mas não foi possível mensurar a influência dessa variável para a espécie, pois, embora todos os animais fossem adultos, eles tinham procedência variada, o que impossibilitou uma estimativa da faixa etária com exatidão.

\section{CONCLUSÕES}

A metodologia empregada neste estudo é viável e reprodutível, contudo recomenda-se a utilização de outro protocolo anestésico, a fim de minimizar os efeitos adversos para a obtenção das imagens ultrassonográficas. Não foram observados artefatos limitantes. Os resultados obtidos neste estudo servirão de referência para estudos futuros e para a clínica de animais silvestres, auxiliando no diagnóstico de afecções oculares.

\section{REFERÊNCIAS}

BANTSEEV, V.; ORIOWO, O.M.; GIBLIN, F.J. et al. Effect of hyperbaric oxygen on guinea pig lens optical quality and on the refractive state of the eye. Exp. Eye Res., v.78, p.925-931, 2004.

BARROS, R.; RODRIGUES, A.C.L.; GUBERMAN, U.C. et al. Mensurações do bulbo ocular e cálculo do poder diotrópico da lente intraocular em miniporcos. Arq. Bras. Med. Vet. Zootec., v.68, p.141-146, 2016.
BATISTA, J.S.; BEZERRA, F.S.B.; AGRA, E.G.D. et al. Efeitos da contenção física e química sobre os parâmetros indicadores de estresse em catetos (Tayassu tajacu). Acta Vet. Bras., v.3, p.92-97, 2009.

CARVAlHO, C.F.; DUPRÉ, A.S.A.; PEREZ, R.B. Ultrassonografia Doppler ocular. In: CARVALHO C.F. Ultrassonografia Doppler em pequenos animais. São Paulo: Roca, 2009. p.152$157 \mathrm{p}$.

CRIVELARO, R.M. Parâmetros oftálmicos em diferentes espécies de cervídeos brasileiros mantidos em cativeiro. 2014. 95f. Dissertação (Mestrado em Cirurgia Veterinária) - Faculdade de Ciências Agrárias e Veterinárias, Universidade Estadual Paulista, Jaboticabal, SP.

DINIZ, A.L.D.; MARON, A.F.; SANTOS, M.C.; SASS, N. Dopplervelocimetria colorida dos vasos orbitais: técnica de exame e anatomia vascular normal. Radiol. Bras., v.37, p.287-290, 2004.

FERNANDES, A.; BRADLEY, D.V.; TIGGES, M. et al. Ocular measurements throughout the adult life span of rhesus monkeys. Investigative Ophthalmol. Visual Sci., v.44, p.2373-2380, 2003.

GAIDDON, J.; ROSOLEN, S.G.; COOK, C.S.; PEIFFER JUNIOR, R. Use of biometry and keratometry for determining optimal power for intraocular lens implant in dogs. Am. J. Vet. Res., v.52, p.781-783, 1991.

GILGER, B.C.; DAVIDSON, M.G.; HOWARD, P.B. Keratometry, ultrasonic biometry, and prediction of intraocular lens power in the feline eye. Am. J. Vet. Res., v.59, p.131-134, 1998.

HARRINGTON, J.T.; McMULLEN, R.J.; CLODE, A.B.; GILGER, B.C. Phacoemulsification and +14 diopter intraocular lens placement in a saddlebred foal. Vet. Ophthalmol., v.16, p.140-148, 2013.

HOFFER, K.J. Biometry of 7,500 cataractous eyes. Am. J. Ophthalmol., v.90, p.360-368, 1980.

LIMA, L.; MONTIANI-FERREIRA, F.; TRAMONTIN, M.H. et al. The chinchilla eye: morphologic observations, echobiometric findings and reference values for selected ophthalmic diagnostic tests. Vet. Ophthalmol., v.13, p.14-25, 2010. 
MIRSHAHI, A.; SHAFIGH, S.H.; AZIZZADEH, M. Ultrasonographic biometry of the normal eye of the Persian cat. Aust. Vet. J., v.92, p.246-249, 2014.

MONTEIRO, E.L.; ALLEMANN, N. Biometria óptica. Arq. Bras. Oftalmol., v.64, p.367-370, 2001.

MONTIANI-FERREIRA, F.; TRUPPEL, J.; TRAMONTIN, M.H. et al. The capybara eye: clinical tests, anatomic and biometric features. Am. Coll. Vet. Ophthalmol., v11, p.386-394, 2008.

MOURA, L.S.; RODRIGUES, R.P.S.; SILVA, A.B.S. et al. Echocardiographic reference ranges for sedated healthy peccaries (Tayassu tajacu, Linnaeus, 1758). Arq. Bras. Med. Vet. Zootec., v.71, p.1-8, 2019.

MUTTI, D.O.; ZADNIK, K.; MURPHY, C.J. The effect of continuous light on refractive error and the ocular components of the rat. Exp. Eye Res., v.67, p.631-636, 1998.

NARVAÉZ, J.; ZIMMERMAN, G.; STULTING, R.D.; CHANG, D.H. Accuracy of intraocular lens power prediction using the Hoffer Q, Holladay 1, Holladay 2, and SRK/T formulas. J. Cataract Refract. Surg., v.32, p.2050-2053, 2006.
RODAS, N.R. Biometria ocular ultrassonográfica e dopplerfluxometria das artérias oftálmica interna e central da retina em macaco-prego (Sapajus spp.). 2014. 93f. Dissertação (Mestrado em Medicina Veterinária e Zootecnia) Universidade Estadual Paulista, Botucatu, SP.

SOUZA, L.P; MERLINI, N.B.; BORTOLINI, Z. et al. Aspectos ultrassonográficos, biometria e dopplerfluxometria ocular no bugio ruivo (Allouata fusca). Pesq. Vet. Bras., v.38, p.10051013, 2018.

SQUARZONI, R.; PERLMANN, E.; ANTUNES, A. et al. Ultrasonographic aspects and biometry of Striped owl's eyes (Rhinoptynx clamator). Vet. Ophthalmol., v.13, p.86-90, 2010.

TONI, M.C.; MEIRELLES, A.E.W.B.; GAVA, F.N. et al. Rabbit's eye globe sonographic biometry. Vet .Ophthalmol., v.13, p.384-386, 2010.

WILLIAMSON, T.H.; LOWE, G.D.; BAXTER, G.M. The influence of age, systemic blood pressure, smoking and blood viscosity on orbital blood velocities. Br. J. Ophthalmol., v.79, p.1722, 1995. 